\title{
Continuous-relief diffractive optical elements for two-dimensional array generation
}

\author{
Michael T. Gale, Markus Rossi, Helmut Schütz, Peter Ehbets, Hans Peter Herzig, and \\ Damien Prongué
}

\begin{abstract}
Continuous surface-relief diffractive optical elements for two-dimensional array generation (fan-out) are designed and fabricated. Separable and nonseparable solutions for the two-dimensional element design are compared. The phase-grating microstructures are generated by laser-beam writing lithography in a single exposure step and converted to nickel shims by electroplating, enabling low-cost replicas to be produced by using laboratory and commercial replication processes. Results are presented for a $9 \times 9$ fan-out diffractive optical element with a measured efficiency of $94 \%$ and an overall uniformity within $\pm 8 \%$; replicas in epoxy have the same efficiency and a uniformity of $\pm 15 \%$.

Key words: Diffractive optical elements, fan-out, laser-beam writing lithography, replication.
\end{abstract}

\section{Introduction}

Space-invariant fan-out optical elements split a single laser beam into an array of quasi plane waves that can be focused by a lens to generate an array of equally intense light spots. Such diffractive optical elements (DOE's) have applications in optical-computing and parallel-processing systems. ${ }^{1}$ The performance requirements include high efficiency and good uniformity of the array. Recent research has concentrated on the realization of multilevel elements ${ }^{2}$ and continuous surface-relief elements. ${ }^{3}$

Continuous surface-relief microstructures represent an attractive alternative to multilevel structures and are, in general, capable of offering higher efficiency. ${ }^{4}$ They can be fabricated by laser-beam writing in photoresist by using a high-precision scanning system with tight control of the resist processing. ${ }^{5}$ Once this technology has been established, it can be effectively used for the fabrication of a wide variety of phase DOE's and other micro-optical components; the writing procedure is a single-step process, so the main effort in fabricating new microstructures would be in the design and programming. The fabrication of replicas by embossing or casting from a metal shim

M. T. Gale, M. Rossi, and H. Schütz are with the Paul Scherrer Institute, Badenerstrasse 569, 8048 Zurich, Switzerland. P. Ehbets, H. P. Herzig, and D. Prongue are with the Institute of Microtechnology, University of Neuchâtel, Rue A.-L. Breguet 2, 2000 Neuchâtel, Switzerland.

Received 29 June 1992.

0003-6935/93/142526-08 $\$ 05.00 / 0$.

(c) 1993 Optical Society of America. enables large numbers of elements to be generated from a single recording.

A laser-beam writing system for the fabrication of continuous-microrelief structures with programmable complex phase profiles has been built at the Paul Scherrer Institute in Zurich (PSIZ). The basic system has been described in an earlier study, together with results for one-dimensional (1-D) fan-out DOE's. ${ }^{5}$ The theory for the design of the optimized continuous phase-transfer function of such 1-D fan-out elements is described in Ref. 6 and is generalized for twodimensional (2-D) design in Ref. 7. Here, nonseparable solutions for a range of 2-D fan-out elements are proposed and compared with separable solutions that are obtained by crossing two 1-D solutions. Detailed experimental results are presented for an original photoresist recording and a replica of a $9 \times 9$ fan-out element, both of which have high efficiency and good uniformity.

\section{Design of Two-Dimensional Fan-Out Elements}

\section{Theory}

The design process for fan-out elements consists of two basic steps: the first leads to high efficiency, and the second optimizes the uniformity of the generated array of light spots with only a minimal decrease in efficiency. In contrast to the optimization of multilevel phase gratings described in Ref. 2, the transfer function of the fan-out element is defined here not in the grating plane but by the array of light spots in the Fourier plane. This approach results in a minimum set of parameters for the exact representation of the 
continuous phase function in the grating plane. The parameters to be optimized are the amplitudes and the phases of an array of point sources.

The desired field distribution in the back focal plane of the lens (Fig. 1) can be written as

$$
U(x, y)=\sum_{m=1}^{M} \sum_{n=1}^{N} A_{m n} \exp \left(i \phi_{m n}\right) \delta\left(x-x_{m}, y-y_{n}\right),
$$

where $A_{m n}$ is the amplitude, $\phi_{m n}$ is the phase, and $\left(x_{m}, y_{n}\right)$ is the position of the ( $m$ th, $n$ th) spot of a 2-D array.

The field distribution $\hat{U}(u, v)$ in the grating plane is related to the field $U(x, y)$ by a Fourier transform:

$$
\begin{aligned}
\hat{U}(u, v) & =\int_{-\infty}^{\infty} \int_{-\infty}^{\infty} U(x, y) \exp [2 \pi i(x u+y v)] \mathrm{d} x \mathrm{~d} y \\
& =\sum_{m=1}^{M} \sum_{n=1}^{N} A_{m n} \exp \left(i \phi_{m n}\right) \exp \left[2 \pi i\left(x_{m} u+y_{n} v\right)\right] .
\end{aligned}
$$

The field $\hat{U}(u, v)$ can be written in terms of magnitude $|\hat{U}(u, v)|$ and phase $\Psi(u, v)$ :

$$
\hat{U}(u, v)=|\hat{U}(u, v)| \exp [i \Psi(u, v)],
$$

where $\Psi(u, v)=\arg (\hat{U})$.

The irradiance distribution $I(u, v)$ in the grating plane can then be expressed as

$$
\begin{aligned}
I(u, v)= & |\hat{U}(u, v)|^{2}=\sum_{m=1}^{M} \sum_{n=1}^{N} A_{m n}^{2}+\sum_{m n} \sum_{m^{\prime} n^{\prime}} A_{m n} A_{m^{\prime} n^{\prime}} \\
& \times \cos \left\{2 \pi\left[u\left(x_{m}-x_{m^{\prime}}\right)+v\left(y_{n}-y_{n^{\prime}}\right)\right]\right. \\
& \left.+\phi_{m n}-\phi_{m^{\prime} n^{\prime}}\right\}
\end{aligned}
$$

where $m \neq m^{\prime}, n \neq n^{\prime}$, and $m+n \leq m^{\prime}+n^{\prime}$.

The first term on the right side of Eq. (4) is constant and equal to the mean object irradiance $\langle I\rangle$. The second term in Eq. (4) describes the variations of the irradiance in the grating plane. These irradiance variations are intermodulation effects and are caused by interference between the waves associated with the $M \times N$ point sources in the focal plane. In

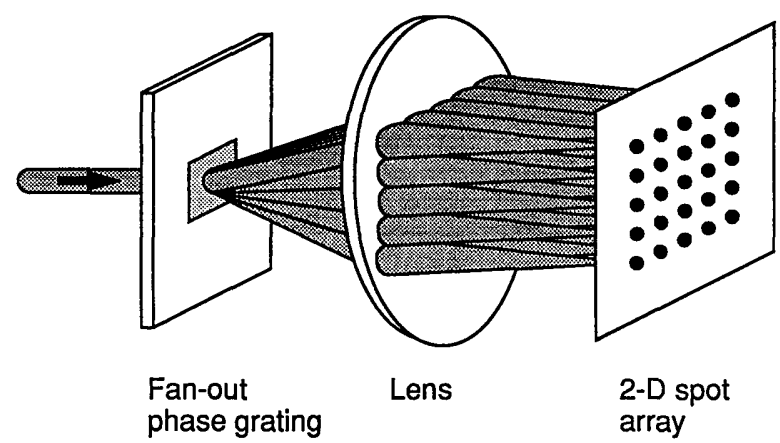

Fig. 1. Readout of fan-out DOE to produce a 2-D array of light spots. the case of regular arrays the expression for the intermodulation terms can be further simplified if the interference terms of equal spatial frequency are collected. We then obtain

$$
\begin{aligned}
I(u, v) & -\langle I\rangle \\
= & 2 \sum_{m n} \sum_{m^{\prime} n^{\prime}} A_{m n} A_{m^{\prime} n^{\prime}} \\
& \times \cos \left\{2 \pi s\left[u\left(m-m^{\prime}\right)+v\left(n-n^{\prime}\right)\right]+\phi_{m n}-\phi_{m^{\prime} n^{\prime}}\right\} \\
= & 2 \sum_{i=1}^{M-1} \sum_{j=1}^{N-1} B_{i j} \cos \left[2 \pi s(u i+v j)+\Phi_{i j}\right]
\end{aligned}
$$

where $i=m-m^{\prime}, j=n-n^{\prime}, s$ is the distance between two neighboring point sources, and $B_{i j}$ and $\Phi_{i j}$ are the amplitudes and the phases, respectively, for one spatial frequency component of the intermodulation terms.

To perfectly reproduce the desired object $U(x, y)$, the fan-out element must have a transfer function proportional to $\hat{U}(u, v)$, which means an intensity transfer function proportional to $I(u, v)$ and a phase transfer function equal to $\exp [i \psi(u, v)]$. In order to reach the highest efficiency a pure phase element is chosen and the intensity transfer function is clipped to $I(u, v)=$ const. Clipping the intensity variations changes the transfer function of the fan-out element and introduces errors at readout. In order to minimize these errors the intermodulation effects in the grating plane have to be reduced as much as possible. The optimization criterion can be formulated as

$\iint\left\{\sum_{i=1}^{M-1} \sum_{j=1}^{N-1} B_{i j} \cos \left[2 \pi s(u i+v j)+\Phi_{i j}\right]\right\}^{2} \mathrm{~d} u \mathrm{~d} v \rightarrow \min$.

Since the intermodulation terms in Eq. (6) have different spatial frequencies, they are orthogonal, and the merit function of the efficiency optimization becomes

$$
\sum_{i=1}^{M-1} \sum_{j=1}^{N-1} B_{i j}^{2} \rightarrow \min
$$

The variables of the efficiency optimization are the phases $\phi_{m n}$ of the point sources, given in Eq. (1), while the amplitudes of the point sources for a uniform fan-out are all equal $\left(A_{m n}=1\right)$. The optimization problem is solved by applying a downhill simplex algorithm. ${ }^{8}$ As a result of the first optimization step, a set of optimum phases $\phi_{m n}{ }^{\text {opt }}$ is obtained. The transfer function of the fan-out element, defined in Eq. (3), is characterized by the phase-transfer function $\exp [i \psi(u, v)]$, calculated for uniform amplitudes and the optimum set of phases. It was found that clipping the minimized intermodulation terms hardly alters the high efficiency, but it reduces the uniformity of the generated array.

In order to improve the uniformity of the fan-out an additional optimization process is used. By iteratively changing the amplitudes of the initial point 
sources $A_{m n}$ slightly to $A_{m n}{ }^{(i)}$, where $i$ is the number of iteration loops, the resulting amplitudes of the generated array can be perfectly balanced. The second optimization process is equivalent to the 1-D case, which is presented in Ref. 6 ; it results in only a slight decrease in the optimized diffraction efficiency from step one. The insertion of both the optimum set of phases $\phi_{m n}{ }^{\text {opt }}$ and the new amplitudes $A_{m n}{ }^{(i)}$ in Eqs. (1) and (2) defines the optimized phase function $\psi(u, v)$ of the fan-out element, which generates a highly uniform array of spots.

\section{Separable and Nonseparable Solutions}

The above theory is used to design nonseparable solutions for different $N \times N$ arrays. In order to treat the most general case, no symmetries of the $N \times N$ arrays are considered. This means that $(N \times N-1)$ variables $\phi_{m n}$ have to be admitted for the efficiency optimization. The phase of one light spot can be kept constant. The number of variables can be a limiting factor for the optimization of large fan-outs. Separable solutions are attractive for generating large $N \times N$ arrays in order to reduce the computing time. In this case the object is described by $U(x, y)=F(x) F(y)$, and thus only the 1-D problem has to be solved. On the other hand, if the 1-D solution of a $N \times 1$ array yields a diffraction efficiency $\eta$, the corresponding 2-D solution of the $N \times N$ array is less efficient, namely $\eta^{2}$. The results for the separable and nonseparable solutions are summarized in Table 1. The efficiencies of the $N \times N$ fan-out are shown after the first and second optimization steps. After the second optimization the unifor-

Table 1. Optimized Efficiencies for 2-D Fan-Out Elements

\begin{tabular}{cccl}
\hline & $\begin{array}{c}\text { Efficiency after } \\
\text { First } \\
\text { Optimization } \\
(\%)^{a}\end{array}$ & $\begin{array}{c}\text { Efficiency after } \\
\text { Second } \\
\text { Optimization } \\
(\%)^{b}\end{array}$ & \multicolumn{1}{c}{$\begin{array}{c}\text { Type of } \\
\text { Solution }\end{array}$} \\
\hline $2 \times 2$ & 64.1 & 64.1 & Separable \\
$2 \times 2$ & 91.8 & 91.8 & Nonseparable \\
$3 \times 3$ & 88.4 & 85.7 & Separable \\
$3 \times 3$ & 94.1 & 93.9 & Nonseparable \\
$4 \times 4$ & 84.6 & 84.4 & Separable \\
$4 \times 4$ & 95.6 & 95.5 & Nonseparable \\
$5 \times 5$ & 96.0 & 84.8 & Separable \\
$5 \times 5$ & 93.6 & 92.6 & Nonseparable \\
$6 \times 6$ & 86.7 & 78.7 & Separable \\
$6 \times 6$ & 94.0 & 93.0 & Nonseparable \\
$7 \times 7$ & 96.0 & 93.7 & Separable \\
$7 \times 7$ & 96.1 & 94.2 & Nonseparable \\
$8 \times 8$ & 92.5 & 92.1 & Separable \\
$8 \times 8$ & 95.1 & 93.6 & Nonseparable \\
$9 \times 9$ & 98.8 & 98.6 & Separable \\
$9 \times 9$ & 98.8 & 98.6 & Nonseparable \\
$10 \times 10$ & 96.4 & 91.0 & Separable \\
$10 \times 10$ & 96.5 & 94.1 & Nonseparable \\
$11 \times 11$ & 97.6 & 95.5 & Separable \\
$11 \times 11$ & 97.6 & 95.5 & Nonseparable \\
\hline
\end{tabular}

${ }^{a}$ First optimization is the efficiency optimization.

'Second optimization is the uniformity optimization. mity error of the generated array is smaller than $0.1 \%$ for all calculated solutions.

As expected, the efficiencies of the nonseparable solutions after the uniformity optimization are always higher than the corresponding separable solutions. The only exception is the $9 \times 9$ fan-out. It provides the highest diffraction efficiency, and in this special case a better solution for a nonseparable design has not been found to our knowledge. The results from Table 1 show that nonseparable solutions are mainly of interest for $N \times N$ fan-outs with $N<9$. For larger 2-D fan-outs the separable solution already provides an efficiency higher than $90 \%$. It can be observed that the efficiencies of the nonseparable solutions decrease less during the second optimization step than those of the separable solutions. The reason is that the intermodulation effects can be better eliminated for the 2-D design.

Symmetries for the phases $\phi_{m n}$ of the 2-D array reduce the number of optimization variables for $2-D$ nonseparable solutions. The obtained efficiencies lie between the two limit values given by the separable and nonseparable asymmetric solutions presented in Table 1.

\section{Fabrication Tolerances}

The realization of these efficient 2-D fan-out elements with the predicted small uniformity error requires accurate fabrication of the surface-relief profile. Figure 2 shows the computed tolerances for the relief profile of the $9 \times 9$ (separable design) array of Table 1 . Errors in the relief profile are represented by an overall linear scale factor in the profile modulation of the microrelief. Whereas a scaling error of $1 \%$ in the amplitude of the microrelief leads to a reduction in efficiency of less than $0.1 \%$, it results in a uniformity error of almost $10 \%$. Achieving good uniformity thus requires accurate control over the microrelief amplitude and profile.

\section{Fabrication by Laser Writing}

A $9 \times 9$ fan-out element of separable design was fabricated by laser-beam writing. The 2-D solution was obtained by crossing two symmetric 1-D solutions, which created an element with a theoretical efficiency of $98.6 \%$ and an almost perfect uniformity.

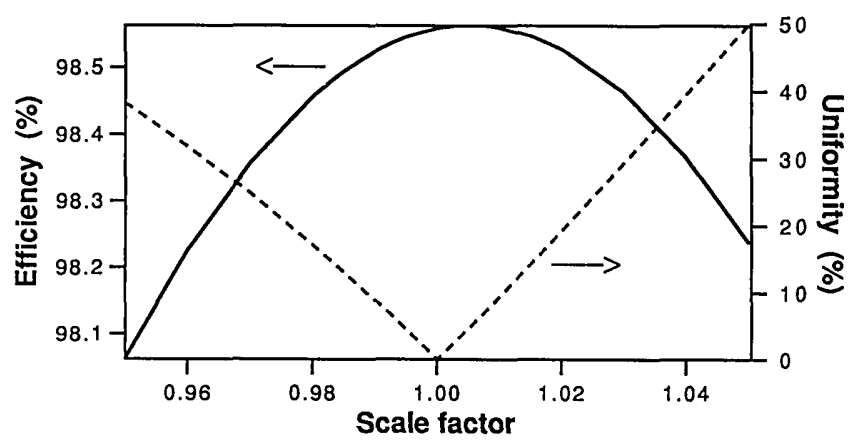

Fig. 2. Computed dependence of the efficiency (solid curve) and the uniformity (dashed curve) upon linear scaling errors in the profile modulation for the $9 \times 9$ fan-out element. 
The numerical values of the optimized 1-D array are given in Table 2. The amplitiudes and phases of the optimum point sources for the 2-D solution are then determined by $A_{m n}=A_{m} A_{n}$ and $\phi_{m n}=\phi_{m}+\phi_{n}$, where $(m, n)=1 \ldots 9$. The unit cell of the optimized phase profile for the $9 \times 9$ fan-out element is shown in Fig. 3.

The optimized phase function for the $9 \times 9$ fan-out element was obtained in photoresist by using the laser-beam writing system at PSIZ, resulting in a continuous surface-relief DOE. The basic writing system and data processing are shown in Fig. 4, and they are described in more detail elsewhere. ${ }^{5,9} \mathrm{~A}$ resist-coated substrate is mounted on a precision air-bearing $x y$ translation table and is scanned under a focused laser spot with a typical raster line spacing of 1 or $2 \mu \mathrm{m}$ and a dynamic rms positioning accuracy of $\sim 150 \mathrm{~nm}$. Exposure data are computed from the desired final microrelief and from the (measured) resist development characteristic. Shipley AZ 1400 resist is used for film thicknesses up to $5 \mu \mathrm{m}$ and is developed in AZ 303 developer to obtain a relatively linear dependence of the developed microrelief as a function of the local exposure. ${ }^{6}$ The resist exposure is controlled by an acousto-optic modulator driven by custom-developed hardware that enables a complete line of 8-bit intensity data to be synchronously clocked out by interferometer pulses that are derived from the table-positioning controller. The $x y$ table scanning speed is $\sim 1 \mathrm{~cm} / \mathrm{s}$, which, combined with the deceleration and acceleration ramping overhead at the end of each line, leads to an exposure time of $\sim 6 \mathrm{~h}$ for a $10 \mathrm{~mm} \times 10 \mathrm{~mm}$ DOE. Development of the exposed resist film results in the required microrelief structure, which can be then further processed to produce a replication shim.

The periodicity of the $9 \times 9$ fan-out element was chosen to be $400 \mu \mathrm{m}$. As the $9 \times 9$ fan-out element has a slowly varying phase function (see Fig. 3), a relatively large writing spot size and considerable overlap of the raster scan lines can be used. This improves the quality of the relief surface by minimizing modulation at the scan-line periodicity ${ }^{10}$ as well as by reducing the sensitivity of the laser writing system to vibrations. A spot size of $8 \mu \mathrm{m}(1 / e$ intensity points) and a raster line spacing of $2 \mu \mathrm{m}$ were used for this fabrication work. One unit cell of the optimized phase function was represented by $200 \times 200$ pixels, and the DOE phase data was converted into microrelief profile by taking into account the refractive index $n$ of the final microstructure material and the readout wavelength $\lambda$.

DOE's were fabricated and evaluated as original

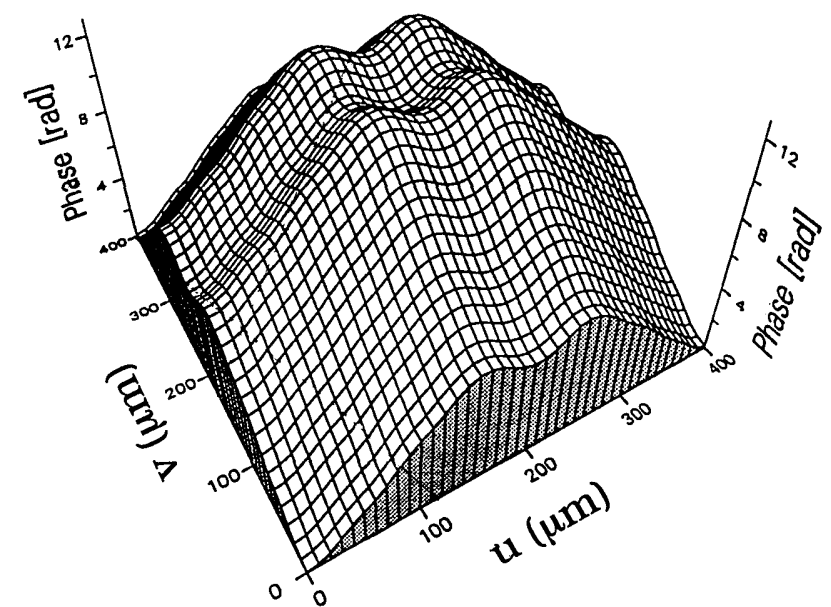

Fig. 3. One period (unit cell) of the optimized phase profile for a $9 \times 9$ fan-out element.

resist recordings ( $n=1.64$ for $\lambda=488 \mathrm{~nm}$ ) and as replicas in Epo-tek 301-2 epoxy ${ }^{11}(n=1.56$ for $\lambda=633$ $\mathrm{nm})$ and polyvinyl chloride (PVC) $(n=1.54$ for $\lambda=633 \mathrm{~nm}$ ). The modulation depth of the resulting microstructures varied between $\sim 1.5$ and $\sim 2.4 \mu \mathrm{m}$. A reproducibility of approximately $\pm 3 \%$ in the profile depth was achieved with careful control of the resist film preparation and the development procedure. Because of the tight fabrication tolerances (see Fig. 2), DOE's were typically fabricated in groups of nine with programmed $1 \%$ or $2 \%$ steps of the modulation amplitude around the design value. The DOE with the best uniformity was then selected from the developed or replicated microstructures.

\section{Replication}

An important feature of DOE's recorded as surfacerelief microstructures is the ability to reproduce the structures by replication techniques such as embossing and casting. For small quantities this can be carried out in the laboratory with relatively simple apparatus; low-cost mass production can benefit from commercial replication processes for holograms and other surface-relief structures.

All replication technologies require the fabrication of a metal shim (a copy of the surface-relief microstructure). Figure 5 illustrates the route from the original recording in photoresist via nickel shims to replicas (further details can be found in Ref. 12). The first step is to fabricate the master (firstgeneration) shim by evaporating a thin silver film ( $\sim 100-\mathrm{nm}$ thick) to form a conductive coating on the photoresist surface. A nickel shim is then produced

Table 2. Optimum Amplitudes $A_{l}$ and Phases $\phi_{l}$ for the Nine-Beam Fan-Out $(i=1 \ldots 9)$

\begin{tabular}{ccccccccccc}
\hline \multirow{2}{*}{$\begin{array}{c}\text { Point-Source } \\
\text { Parameter }\end{array}$} & 1 & 2 & 3 & 4 & 5 & 6 & 7 & 8 & 9 \\
\cline { 2 - 10 }$n$ & 1.059 & 0.957 & 0.987 & 0.998 & 1.022 & 0.998 & 0.987 & 0.957 & 1.059 \\
$A_{i}$ & 1.772 & 0.135 & 3.887 & 2.455 & 3.142 & 2.455 & 3.887 & 0.135 & 1.772 \\
$\phi_{i}(\mathrm{rad})$ & &
\end{tabular}




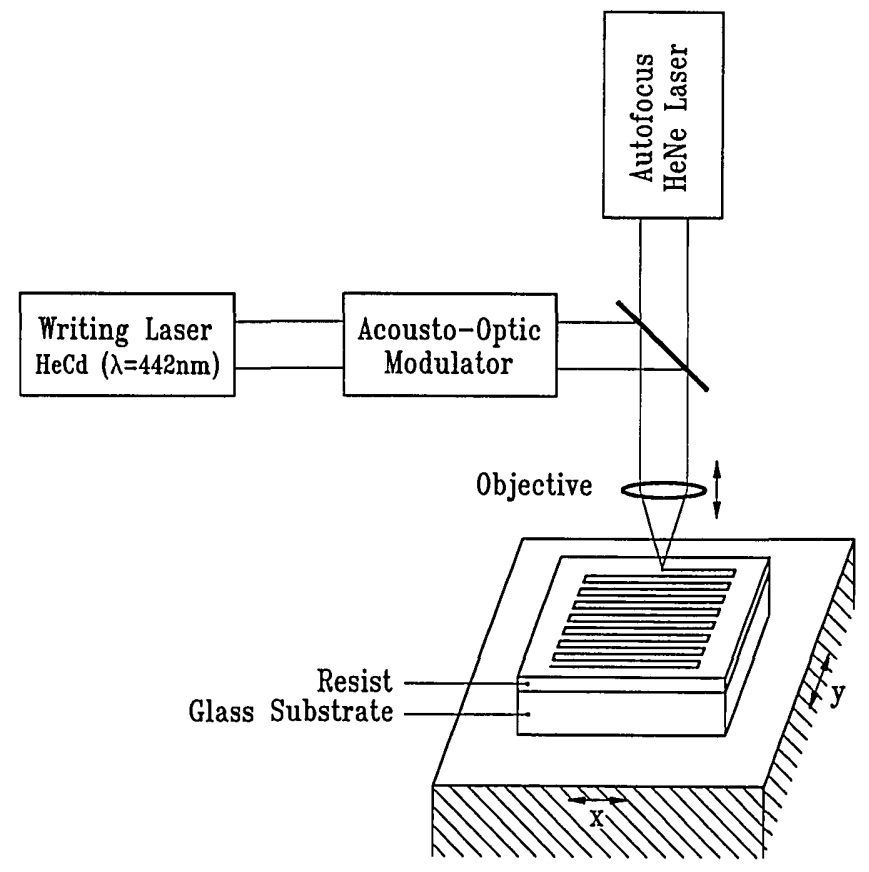

Precision air-bearing xy translation stage

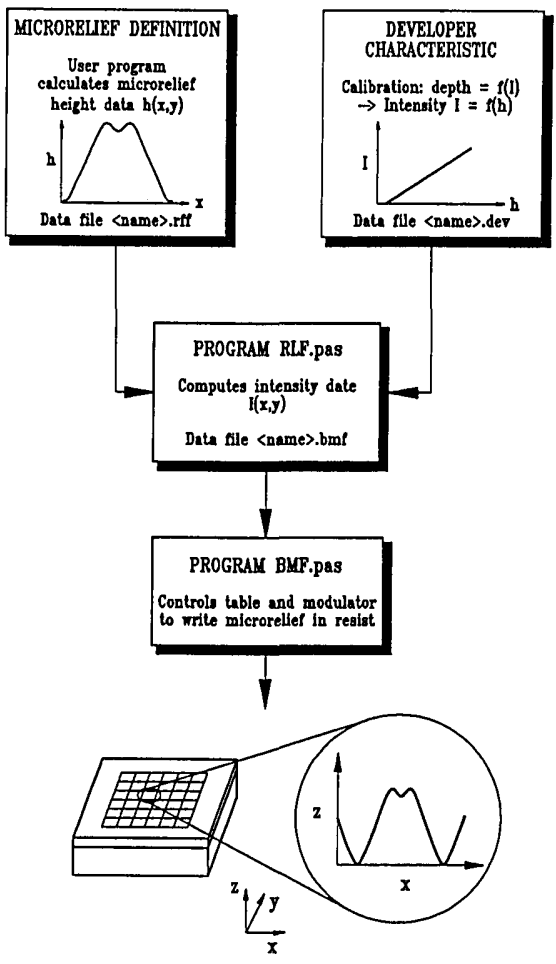

Fig. 4. Schematic of the software and hardware in the laser-beam writing system.

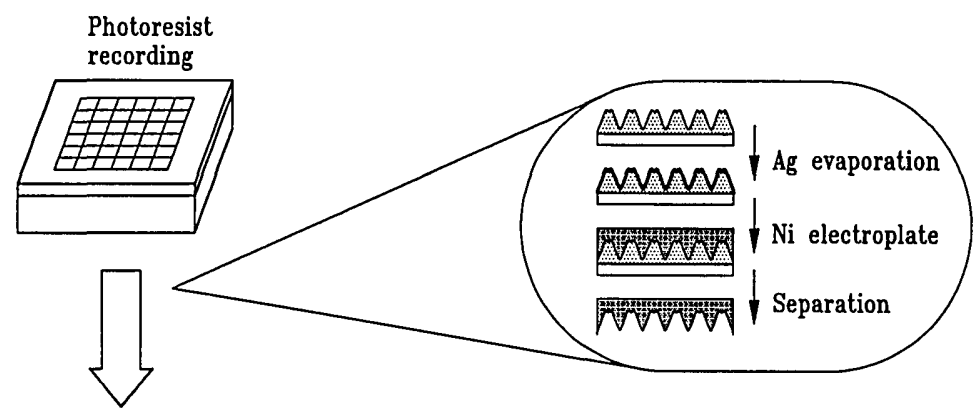

HOT EMBOSSING (Stamping)
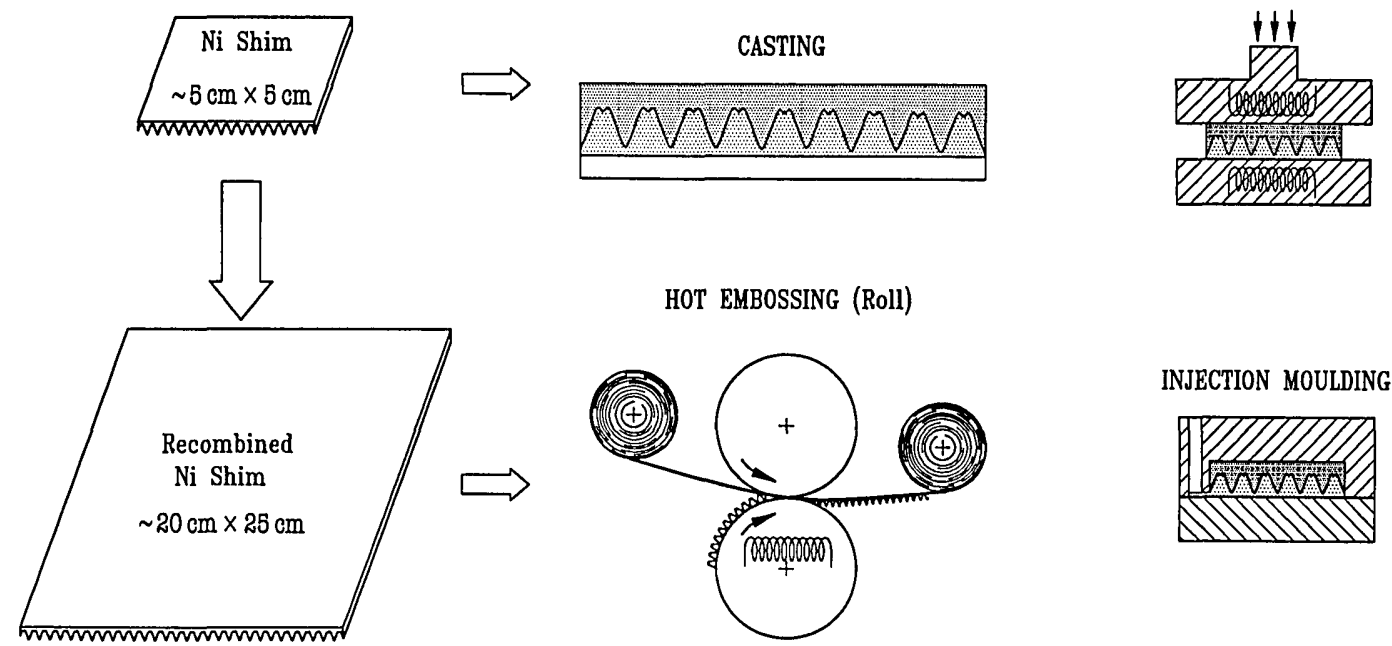

HOT EMBOSSING (Roll)

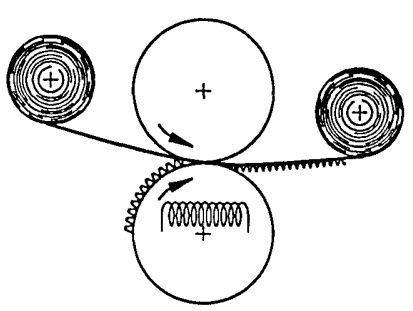

INJECTION MOULDING

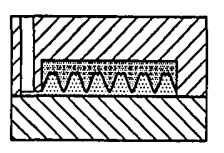

Fig. 5. Fabrication of replicas from an initial photoresist DOE recording. Casting and hot stamping are relatively simple processes suitable for replication in the laboratory. Roll embossing and injection moulding are industrial replication technologies suited to low-cost mass production. 


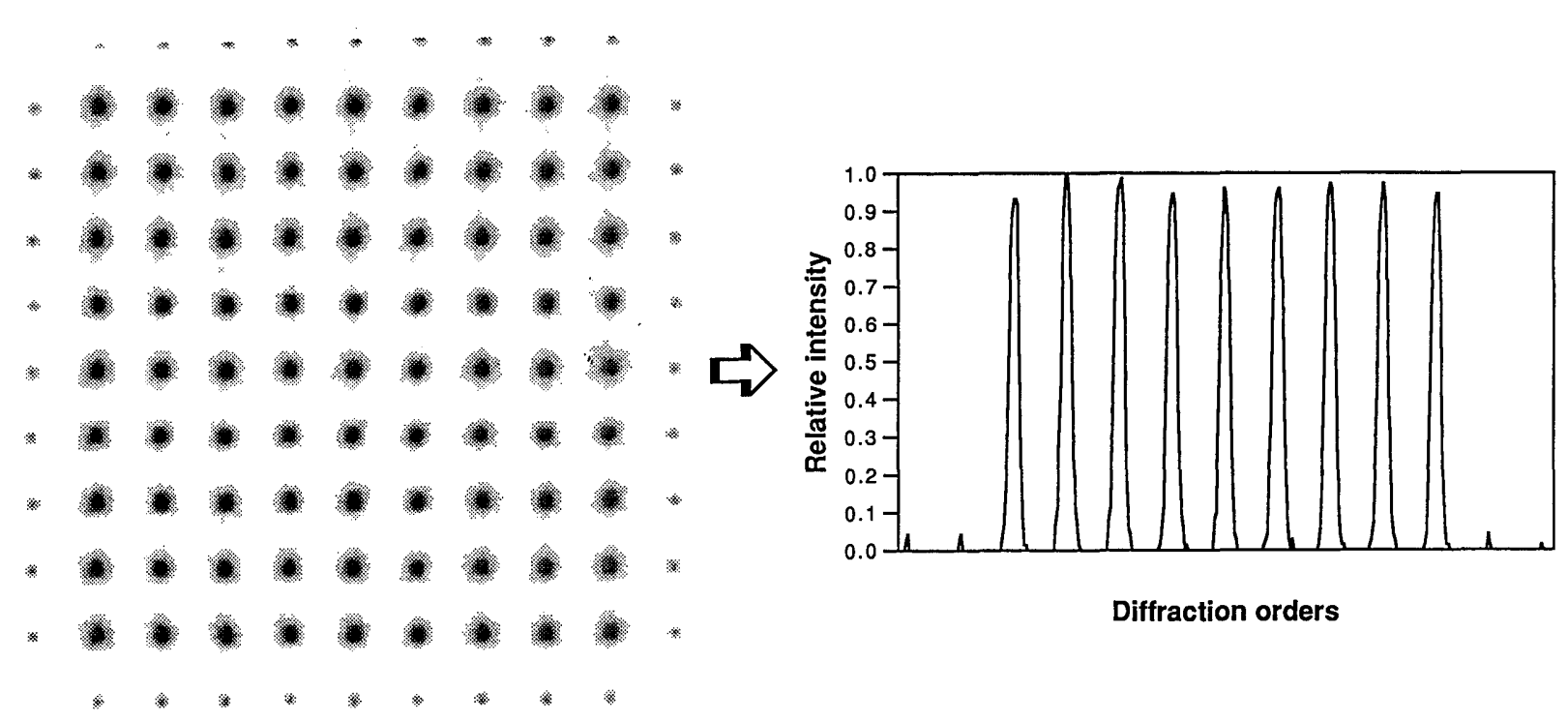

Fig. 6. Fan-out DOE in photoresist: measured spot pattern and intensities along the central line.

by electroplating $\mathrm{Ni}$ to a typical thickness of $60-100$ $\mu \mathrm{m}$ (the photoresist microstructure is destroyed on separation from this metal copy). Subsequent generations of shims are obtained by passivating the shim surface in dichromate and electroplating a further copy. In general, third-generation shims are most suitable for the replication process, since the profile polarity is correct for producing a replica identical to the original photoresist recording, and multiple copies can be generated from a single second-generation shim without risking the first-generation master.

The main approaches for replication (see Fig. 5) are the following:

Casting. High-quality replicas can be fabricated in the laboratory by casting into a thin film of curable epoxy (e.g., Epo-tek 301-2) coated onto a glass substrate. Curing is typically thermal, although UVcurable materials can also be used.

Embossing. Both flat-bed (stamping) and continuous-roll embossing are currently used for the commercial replication of surface-relief microstructures such as security holograms and diffractive packaging film.12,13 Roll embossing into thermoplastic film (such as PVC) is capable of achieving fast replication (up to $1 \mathrm{~m}^{2} / \mathrm{s}$ ) at low cost (down to $\$ 1 / \mathrm{m}^{2}$ ). Current hot-embossing technology has been developed for microstructures up to $\sim 1 \mu \mathrm{m}$ in amplitude; successful replication of deeper microstructures requires careful optimization of the materials and process conditions.

Injection moulding. Replication using injectionmoulding processes such as those used for the production of compact disks have been shown to produce high-quality replicas of microrelief structures in poly(methyl methacrylate). ${ }^{14}$ Although this technology probably has the higher costs per unit area, the basic process is well established for compact disk production and is capable of producing excellent replicas.

The current PSIZ laser writing system produces recordings and shims of typically $5 \mathrm{~cm} \times 5 \mathrm{~cm}$ in size. These are used for laboratory replication tests and
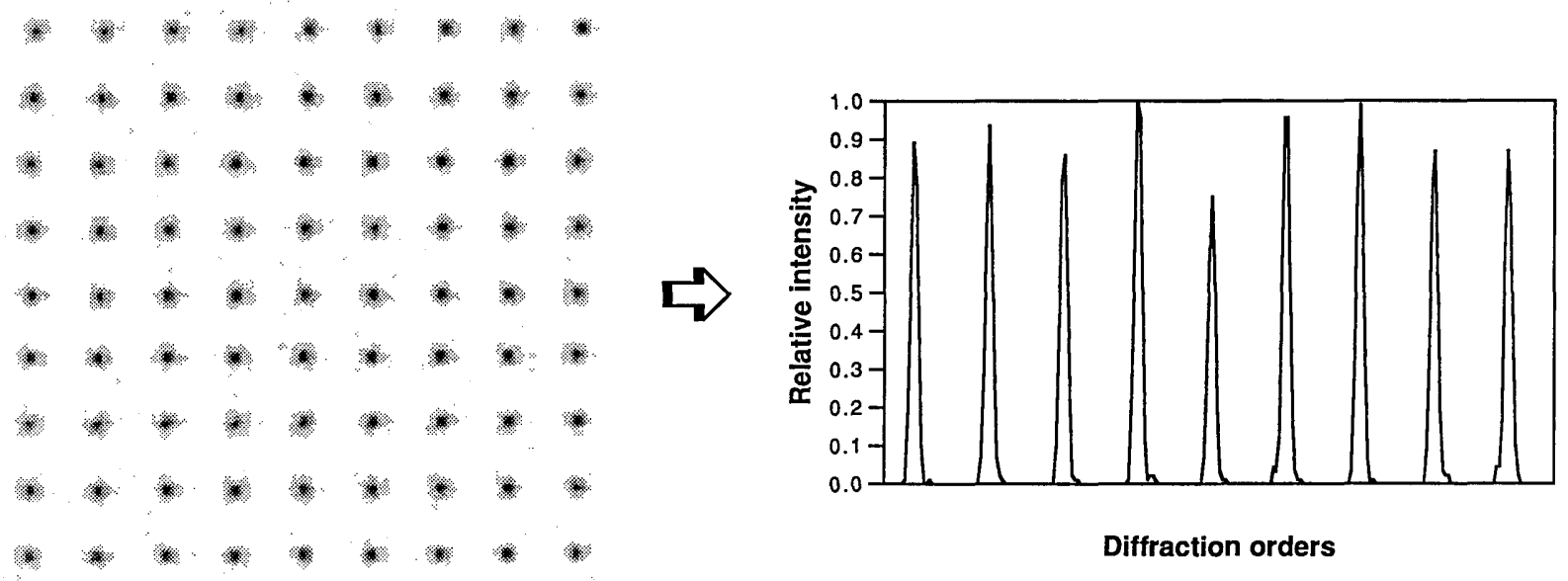

Fig. 7. Same as Fig. 6 but for the fan-out DOE replica in epoxy. 


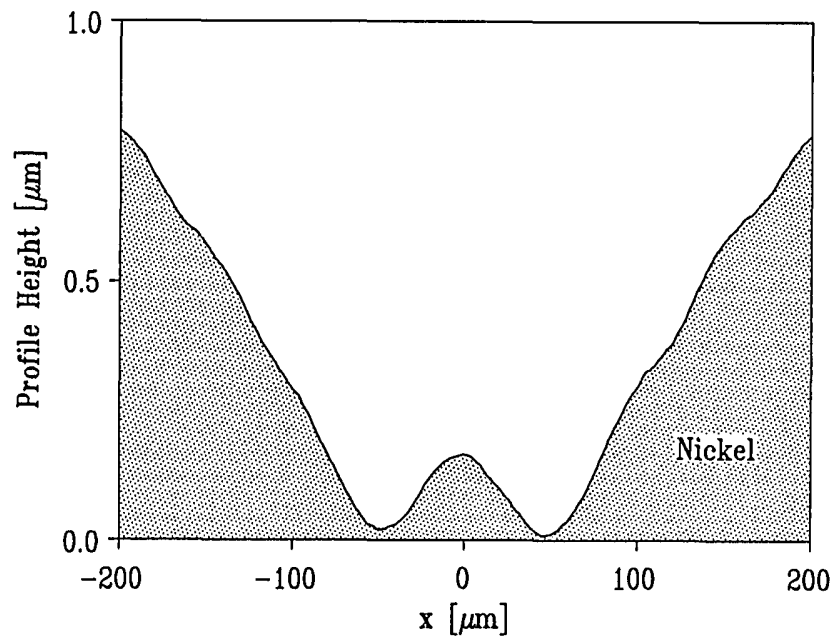

(a)

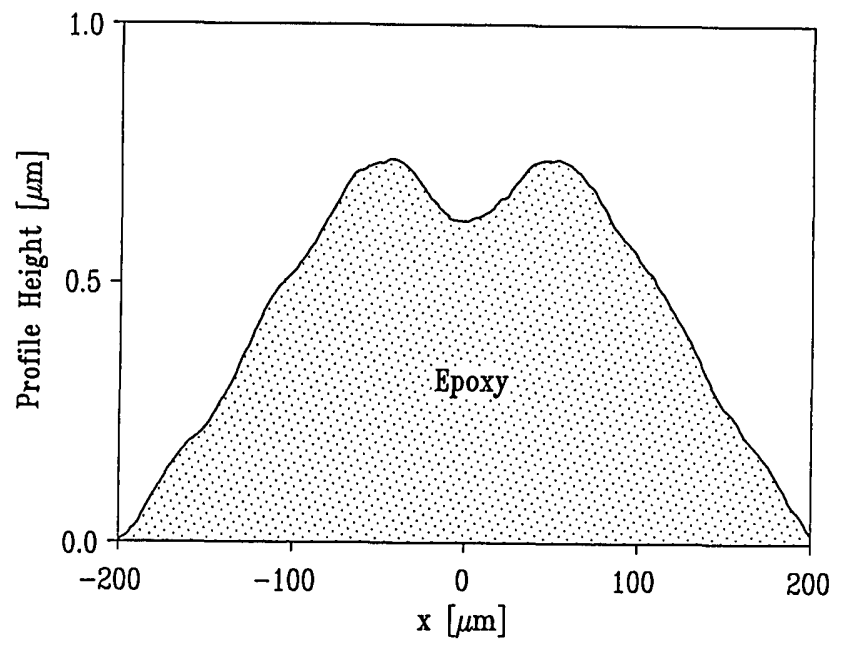

(b)

Fig. 8. Profile measurements of (a) a nickle shim and (b) the replica in epoxy. The slight difference in the profiles and modulation amplitudes are due to different measurement line positions.

small volume production by casting or flat-bed embossing. Commercial equipment for flat-bed and roll embossing of holographic microstructures requires shims of typically $20 \mathrm{~cm} \times 25 \mathrm{~cm}$ in size. For test embossings in PVC, such production shims were generated by recombination from the smaller shims produced from the laser-written microstructure. ${ }^{15}$

\section{Results}

DOE's with $9 \times 9$ fan-out (separable design) were fabricated in photoresist by laser-beam writing and converted to nickel shims by electroplating. Replicas were then produced by casting into epoxy on glass and by embossing into PVC sheet. Large, recombined shims are currently being used for test replication in PVC on a commercial embossing system.

Figure 6 shows the image recorded by the CCD camera at the back focal plane of the lens (cf. Fig. 1) for the reconstruction from a $9 \times 9$ fan-out DOE in photoresist illuminated by a collimated argon laser beam $(\lambda=448 \mathrm{~nm})$. The figure shows the image of the reconstructed spots and the intensities in the

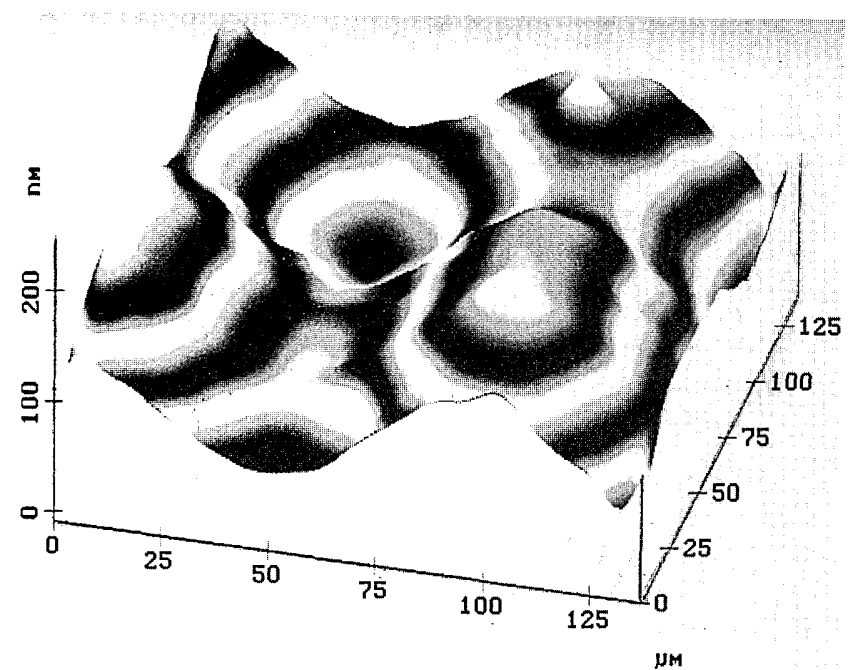

Fig. 9. Atomic force microscope image showing a 2-D profile at the center of a fan-out DOE unit cell.

central row (which includes the zero order). The spot intensities were measured by using a detector with an aperture diameter equal to one fifth the spot spacing. Uniformity is defined as $\left(I_{\max }-I_{\min }\right) /$ $\left(I_{\max }+I_{\min }\right)$, where $I_{\max }$ and $I_{\min }$ are the maximum and minimum spot intensities. The efficiency values given are the sum of the spot intensities as a fraction of the total light transmitted through the DOE. The measurements for this DOE obtained an efficiency of $94 \%$ and a uniformity of $\pm 8 \%$ over the whole $9 \times 9$ array. Within one line or one row the uniformity was better than $\pm 5 \%$.

Corresponding results for a replicated fan-out DOE in epoxy are shown in Fig. 7. The structure was designed for a refractive index $n=1.56$ (Epo-tek $301-2$ ) and a readout at $\lambda=633 \mathrm{~nm}$. The efficiency is also $\sim 94 \%$, but the uniformity is somewhat lower, $\sim \pm 15 \%$, which can be traced to errors in the recording process leading to a relief amplitude that is deeper than the design value (cf. Fig. 2). Line surface profile measurements for a nickel shim and the replicated DOE in epoxy are shown in Fig. 8 (the slightly different modulation amplitudes are due to different measurement line positions). An atomic force microscope profile measurement of the central $140 \mu \mathrm{m} \times 140 \mu \mathrm{m}$ of the $400 \mu \mathrm{m} \times 400 \mu \mathrm{m}$ unit cell is shown in Fig. 9.

\section{Conclusions}

The design of continuous-relief DOE microstructures for 2-D array generation has been investigated, and the performance of separable and nonseparable solutions has been compared. Nonseparable solutions are of interest mainly for $N \times N$ format with $N<9$. For larger 2-D fan-out structures the separable solution already provides a satisfactory efficiency of $>90 \%$.

Fan-out DOE's have been fabricated by laser-beam writing in photoresist. Replicas were produced from the photoresist recording by electroplating a nickel shim and hot embossing into thermoplastic film or by 
casting in optical epoxy. Replicated $9 \times 9$ fan-out DOE's fabricated with the latter approach showed an excellent efficiency of $94 \%$ and a uniformity of $\pm 15 \%$. The technology enables a wide variety of DOE microstructures to be fabricated by using a single-step writing process and reproduced in large or small volume at reasonable cost.

The authors are grateful to C. Appassito, G. K. Lang, and J. M. Raynor for their contributions in developing the control hardware and software for the laser writing system and to J. S. Pedersen for electroplating the replication shims. We also thank $R$. Dändliker, K. Knop, and R. E. Kunz for numerous fruitful discussions and ideas. This work was supported in part by the Board of the Swiss Federal Institutes of Technology.

\section{References and Notes}

1. N. Streibl, "Beam shaping with optical array generators," J. Mod. Opt. 36, 1559-1573 (1989).

2. J. Turunen, J. Fagerholm, A. Vasara, and M. R. Tagizadeh, "Detour-phase kinoform interconnects: the concept and fabrications considerations," J. Opt. Soc. Am. 7, 1202-1208 (1990).

3. H. P. Herzig, D. Prongué, and R. Dändliker, "Design and fabrication of highly efficient fan-out elements," Jpn. J. Appl. Phys. 29, L1307-L1309 (1990).

4. D. Daley, S. M. Hodson, and M. C. Hutley, "Fan-out gratings with a continuous profile," Opt. Commun. 82, 183-187 (1991).
5. M. T. Gale, G. K. Lang, J. M. Raynor, H. Schütz, and D. Prongué, "Fabrication of kinoform structures for optical computing," Appl. Opt. 31, 5712-5715 (1992).

6. D. Prongué, H. P. Herzig, R. Dändliker, and M. T. Gale, "Optimized kinoform structures for highly efficient fan-out elements," Appl. Opt. 31, 5706-5711 (1992).

7. P. Ehbets, H. P. Herzig, D. Prongué, and M. T. Gale, "Highefficiency continuous surface-relief gratings for two-dimensional array generation," Opt. Lett. 17, 908-910 (1992).

8. W. H. Press, B. P. Flannery, S. A. Teukolsky, and W. T. Vetterling, Numerical Recipes in Pascal (Cambridge U. Press, Cambridge, 1989).

9. M. T. Gale, G. K. Lang, J. M. Raynor, and H. Schütz, "Fabrication of micro-optical components by laser beam writing in photoresist," in Micro-Optics II, A. M. Scheggi, ed., Proc. Soc. Photo-Opt. Instrum. Eng. 1506, 65-70 (1991).

10. M. T. Gale and K. Knop, "The fabrication of fine lens arrays by laser beam writing," in Industrial Applications of Laser Technology, W. F. Fagan, ed., Proc. Soc. Photo-Opt. Instrum. Eng. 398, 347-353 (1983).

11. Product of Epoxy Technology, Inc., Billerica, Mass.

12. B. Kluepfel and F. Ross, eds., Holography Market Place (Ross, Berkeley, Calif., 1991).

13. M. T. Gale, "Diffractive microstructures for security applications," IEE Conf. Publ. London 342, 205-209 (1991).

14. A. Neyer, T. Knoche, P. Dannberg, and L. Müller, "New fabrication technology for polymer optical waveguides," in Integrated Photonics Research, Vol. 10 of 1992 OSA Technical Digest Series (Optical Society of America, Washington, D.C., 1992), paper WB2-1.

15. The recombination and PVC embossings were carried out by 3D Ltd., Unterägeri, CH-6314, Switzerland. 